\title{
Strength Variation of Parachute Joints
}

\author{
Catherine Mollmann* \\ Airborne Systems North America, Pennsauken, NJ, 08109
}

\begin{abstract}
A parachute joint is defined as a location where a component is sewn or connected to another component. During the design and developmental phase of a parachute system, the joints for each structural component are isolated and tested through a process called seam and joint testing. The objective of seam and joint testing is to determine the degradation on a single component due to interaction with other components; this data is then used when calculating the margin of safety for that component.

During the engineering developmental phase of CPAS (Capsule Parachute Assembly System), the parachute system for the NASA Orion Crew Module, testing was completed for every joint of the six subsystems: the four parachutes (main, drogue, pilot, and FBCP [forward bay cover parachute]), the retention release bridle, and the retention panels. The number of joint tests for these subsystems totaled 92, which provides a plethora of data and results for further analysis.

In this paper, the data and results of these seam and joint tests are examined to determine the effects, if any, of different operators and sewing machines on the strength of parachute joints. Other variables are also studied to determine their effect on joint strength, such as joint complexity, joint strength magnitude, material type, and material construction. Findings reveal that an optimally-run seam and joint test program could result in an increased understanding of the structure of the parachute; this should lead to a parachute built with optimal components, potentially saving system weight and volume.
\end{abstract}

\section{Nomenclature}

$\begin{array}{ll}A L & =\text { applied load } \\ C P A S & =\text { Capsule Parachute Assembly System } \\ D F & =\text { design factor } \\ E S & =\text { element strength } \\ F B C & =\text { forward bay cover } \\ F B C P & =\text { forward bay cover parachute } \\ M S & =\text { margin of safety } \\ N P & =\text { number of plies } \\ u & =\text { joint efficiency }\end{array}$

\section{Introduction}

A seam and joint testing program is typically instituted during the design and developmental phase of a parachute system. During this testing program, the strengths of all of the structural parachute components are examined at locations where they are sewn to or interact with other components. On CPAS, and as a standard Airborne Systems practice, each structural joint on a parachute is isolated in a single joint test. The strength of a set of joint samples, which are constructed using the same steps and materials as the actual parachute joint, is compared to the strength of a set of control samples, the base material. This process is covered in detail in "Determination of Parachute Joint Factors using Seam and Joint Testing." 2

Each joint test on CPAS consisted of ten joint samples and five control samples. Per CPAS contract requirements, five of the joint samples were constructed by one set of operators using one set of sewing machines, while the other five were constructed by another set of operators using another set of sewing machines. This was completed in order to understand and envelop any potential workmanship or performance differences that different operators and/or sewing machines may have caused. Requiring two sets of operators and sewing machines requires more schedule and

\footnotetext{
* Production Engineer, Engineering, 5800 Magnolia Ave, Pennsauken, NJ, 08109, AIAA Member.
} 
manufacturing time. Most joint sample sets are made using more than a single operator and/or sewing machine, as they can consist of dozens of manufacturing steps and sewing with different machines and thread types. With the CPAS requirement to split the ten joint samples into two sets, twice the number of operators had to be trained and supervised and twice the number of sewing machines had to be identified and set up for each joint test. Although two sets of operators were then potentially working in parallel, no time is saved on an overall time basis. Results from the CPAS seam and joint testing program can be used to investigate the necessity of this requirement.

While considerable consideration has been given to enveloping the effects of different operators and sewing machines, other factors also contribute to variations in joint strength. Throughout this paper, an examination of these other variables is studied. Some examples of factors which have a large impact on joint strength variation include the complexity of the joint, the magnitude of the base material strength, and joints where stitches break instead of base material. Effects on strength due to material type (e.g. Kevlar ${ }^{\circledR}$ vs. Nylon) and construction type (e.g. weave vs. braid) were also investigated. Recommendations are given at the end of the paper for consideration in future joint designs and for seam and joint testing programs.

\section{Overview of Analysis}

\section{A. Definition of Terms}

The major terms used in this paper are defined below. The explicit quantities included in the definitions are specific to CPAS, though the definitions hold true for all seam and joint testing campaigns:

- Joint test: represents a single parachute structural joint. A joint test consists of ten total joint samples (or two test sets) as well as five control samples. All material in the ten joint samples and five control samples are constructed from the same lots of materials using the same manufacturing steps.

- Test set: five joint samples that were constructed by the same operators on the same sewing machines using the same material and manufacturing steps. There are two test sets per joint test.

- Joint sample: a single representation of a joint, which replicates the design and parts list from the actual parachute. There are five joint samples in each test set and ten joint samples in each joint test.

- Control sample: a single length of the base material that is used for comparison to the joint samples. There are five control samples in each joint test. Control samples provide a comparison to the joint samples, as opposed to using the minimum specification strength of that material.

Figure 1 gives a visual overview of the relationship between the above terms:

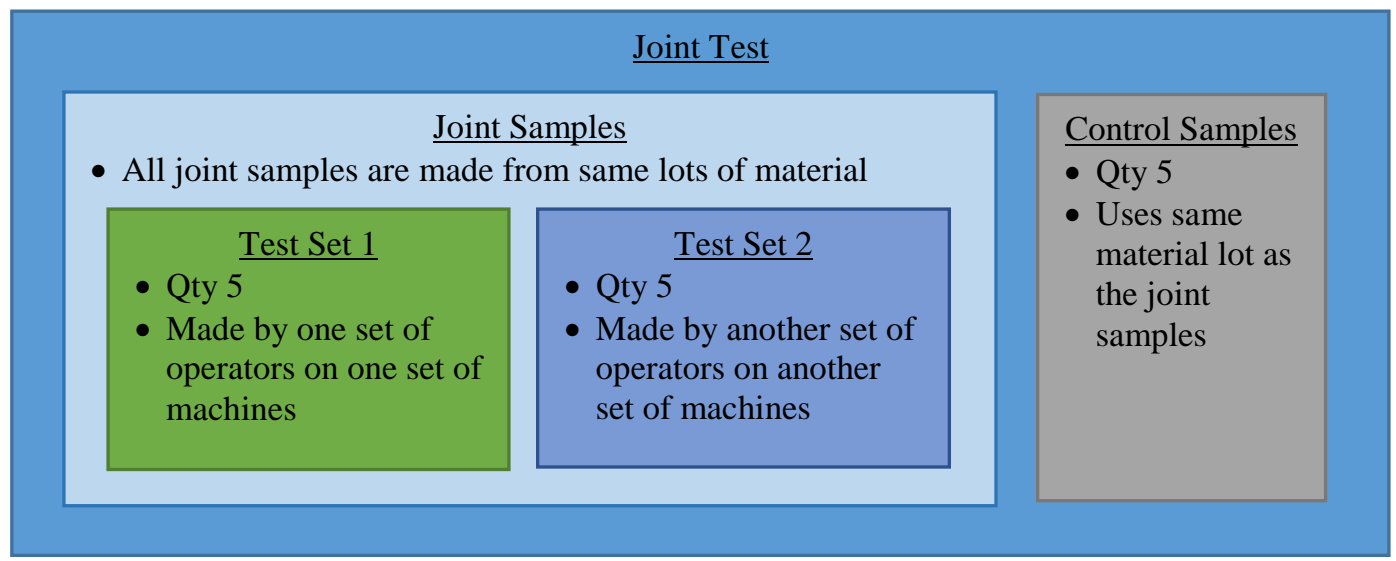

Figure 1. Joint Test Configuration

\section{B. Relationship to Joint Efficiency and Margin of Safety}

Joint tests are designed to isolate each joint in order to enable individual strength analysis and potential improvements. After destructive pull testing of both the joint samples and the control samples, an efficiency is calculated. This efficiency represents how much the base component is degraded due to sewing or interactions with other components. The efficiency, called the joint efficiency, is calculated using Eq. (1), per CPAS and Airborne standards: 


$$
\text { Joint Efficiency }(u)=\frac{\text { (Joint Test Average }- \text { Joint Test Standard Deviation })}{(\text { Control Average })(\text { Number of Plies })}
$$

The number of plies refers to the minimum number of layers along the entire length of the joint sample, which is the number of members sharing load during the tensile test; this assumes that the layers share the load equally. The joint efficiency $(u)$ is used along with several other degradation factors in the calculation of the design factor, as shown in Eq. $(2)^{1}$ :

$$
\text { Design Factor }=D F=\frac{m * s * c}{u * e * o * k * t * a} * S F
$$

" $u$ " refers to the joint efficiency; since there are typically several joint efficiencies calculated for a single component, the minimum efficiency is used to calculate the design factor for each component. All of the other variables in Eq. (2) refer to other degradation factors such as safety factor (SF), temperature (t), abrasion (e), asymmetry (s), etc. This design factor is then used in conjunction with the maximum applied load (AL) and the minimum strength of the base material (ES) to calculate the margin of safety (MS) for each structural component, as shown in Eq. (3):

$$
\text { Margin of Safety }=M S=\frac{(\text { Element Strength })(\text { Number of Plies })}{(\text { Applied Load })(\text { Design Factor })}-1=\frac{E S * N P}{A L * D F}-1
$$

The margin of safety is a bounding calculation because it assumes that the maximum applied load (AL) occurs at the same time as all of the degradation factors (DF), when this is not usually the case. It also only takes credit for the minimum acceptable material strength per the specification (ES), even though material is always constructed stronger than the minimum. Through examination of the previous three equations, it is apparent that when the joint efficiency decreases, the design factor increases and the margin of safety decreases. All other variables being equal, lower joint efficiencies necessitate a stronger (and heavier) material in order to achieve positive margin for a particular structural component.

As mentioned in the previous section, control samples are used in order to realize the actual strength loss of the base material. Material specifications provide a minimum allowable strength for the material and material is always constructed above this minimum, often by as much as $20 \%$. Comparing tensile test results to the minimum acceptable strength in Eq. (1) would result in a higher joint efficiency than if the strength of the control samples were used. This would introduce the risk that in the future, a parachute could be built from material closer in strength to the minimum than the joint samples were; this would result in a lower parachute strength than the minimum calculated in the structural analysis (which could result in a negative margin of safety).

The joint efficiency, as calculated in Equation 1, is also often referred to as the standard efficiency. The joint/standard efficiency subtracts one standard deviation from the joint test results, which is intended to account for most of the incidental variation in strength between sewn joints. Another efficiency is the mean efficiency. See Eq. (4):

$$
\text { Mean Efficiency }=\frac{(\text { Joint Test Average })}{(\text { Control Average })(\text { Number of Plies })}
$$


What differentiates the mean efficiency and standard (joint) efficiency is the inclusion or exclusion of the joint test standard deviation. The purpose of including the standard deviation in the joint efficiency calculation is to ensure that variations in joint sample strengths within a joint test are understood and incorporated. As a result of its inclusion, the standard efficiency is always lower than the mean efficiency. This difference is typically $2-6 \%$, as seen in Table 1 below, where this data is taken from all 92 of the CPAS seam and joint tests:

Table 1. Difference between Mean and Standard Efficiency

\begin{tabular}{|c|c|}
\hline \multicolumn{2}{|c|}{ (Mean Efficiency) - (Standard Efficiency) } \\
\hline Minimum & $1.0 \%$ \\
\hline Maximum & $10.0 \%$ \\
\hline Average & $4.0 \%$ \\
\hline Standard & $1.7 \%$ \\
\hline Average - Standard Deviation & $2.3 \%$ \\
\hline Average + Standard Deviation & $5.7 \%$ \\
\hline
\end{tabular}

The standard deviation of the joint samples is driven by the differences in the breaking strengths within the entire test set. As the standard deviation increases, the joint efficiency decreases. The variables and types of tests that increase this standard deviation are examined in this paper.

\section{Overview of Data and Analysis Methods}

As previously stated, there are six CPAS subsystems: main parachutes, drogue parachutes, pilot parachutes, FBCPs, retention release bridles, and retention panels. Combining the joint tests from all six subsystems, this results in 92 total tests, or $92 * 2=184$ total test sets. The data from these joint tests ${ }^{3}$, from both the joint samples and control samples, are analyzed in this paper.

\section{Overview of Operators}

Many of the joint tests were manufactured using more than one operator and one sewing machine; this makes it difficult to measure the difference between particular operators and particular machines. For example, one set of joint samples could be constructed by operators A and B. Another set of joint samples for another joint test could be constructed by operators A and C. This makes it difficult to isolate the performance of operator A from the existing data. Therefore, differences between entire test sets within a single joint test are examined.

As the CPAS seam and joint testing campaign encompasses almost 100 joint tests manufactured and tested over five years, a variety of operators aided in the manufacturing. Table 3 below illustrates the number of operators and the frequency of how often they contributed to manufacturing a test set ${ }^{3}$ :

Table 2. Frequency of Operators

\begin{tabular}{|c|c|c|}
\hline $\begin{array}{c}\text { Number of Test Sets } \\
\text { Manufactured }\end{array}$ & $\begin{array}{c}\text { Number of } \\
\text { Operators }\end{array}$ & $\begin{array}{c}\text { Percentage of } \\
\text { Operators }\end{array}$ \\
\hline $\mathbf{1}$ & 7 & $25 \%$ \\
\hline $\mathbf{2 - 6}$ & 8 & $29 \%$ \\
\hline $\mathbf{7 - 1 9}$ & 9 & $32 \%$ \\
\hline $\mathbf{2 0 - 3 9}$ & 1 & $4 \%$ \\
\hline $\mathbf{2 4 0}$ & 3 & $11 \%$ \\
\hline All & 28 & $100 \%$ \\
\hline
\end{tabular}

Table 2 shows that of the 28 total operators, the majority (15) aided in the manufacturing of only six or fewer test sets. There were three operators that contributed to 40 or more test sets, with one operator working on 63 test sets. While these three operators worked on a large proportion of the test sets, it can be seen that there was reasonably high variation in operators across the entire set of joint tests. 


\section{Percent Difference}

Two methods of analysis are used in this paper to compare joint test data. The primary tool is percent difference. Percent difference, calculated in Eq. (5) below, is used to compare the average breaking strength of each test set to that of the overall joint test. As there are two test sets per joint test, each CPAS joint test results in two separate percent differences.

$$
\text { Percent Difference }=100 *\left|\frac{\text { Difference }}{\text { Average }}\right|=100 *\left|\frac{\text { Test Set Average }- \text { Joint Test Average }}{\text { Average of Test Set and Joint Test }}\right|
$$

\section{Confidence Level and Critical Z-Score}

The second method of comparison is the confidence level and the z-score. A z-score is a measure of how many standard deviations a joint sample value is away from the average. See Eq. (6):

$$
z_{\text {score }}=\left|\frac{\text { Value }- \text { Average }}{\text { Standard Deviation }}\right|=\left|\frac{\text { Joint Sample Value }- \text { Joint Test Average }}{\text { Joint Test Standard Deviation }}\right|
$$

The lower the z-score, the closer the individual joint sample value is to the joint test average. The desired confidence level determines the critical z-score (or vice versa). The confidence level, expressed as a percentage, is how likely each joint sample is to be below the critical z-score. Therefore, a higher confidence level necessitates a higher critical z-score.

\section{Results of Operator- and Sewing Machine-Dependency Analysis}

\section{A. Percent Difference}

\section{Overall Test Data}

The percent difference was calculated to compare the average breaking strength for each test set to that of the overall joint test. As the two test sets were constructed using the same manufacturing steps and the same lots of materials, these results primarily compare the differences between the sets of operators and sewing machines. The left image of Figure 2 gives the percent difference for the test sets of all six CPAS subsystems, while the right image of Figure 2 combines all of the subsystems together:
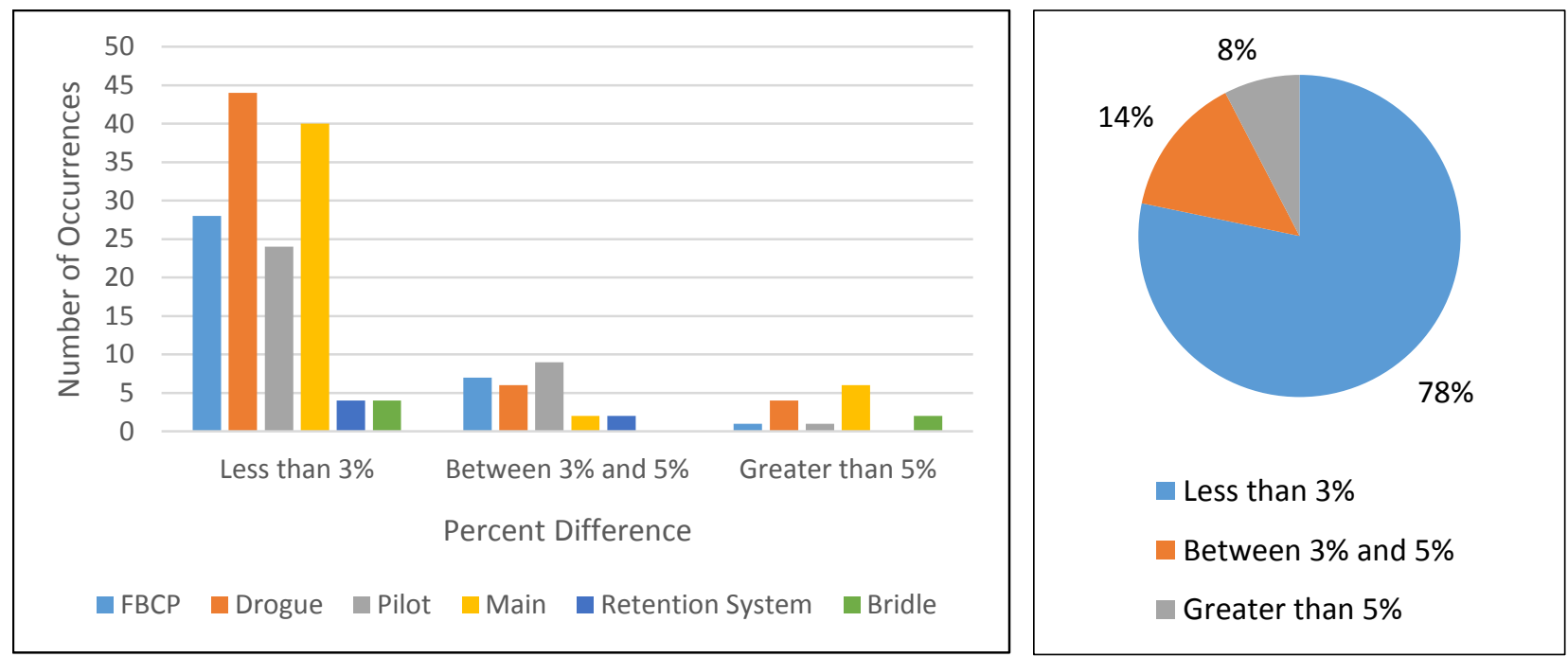

Figure 2. Percent Difference among CPAS Subsystems

While the majority of the test sets had a percent difference less than 3\%,22\% were greater than $3 \%$. These test sets with high percent differences are discussed more in Section IV. 
2. Split by Sub-System

Figure 3 gives the percent difference for each of the six different subsystems:
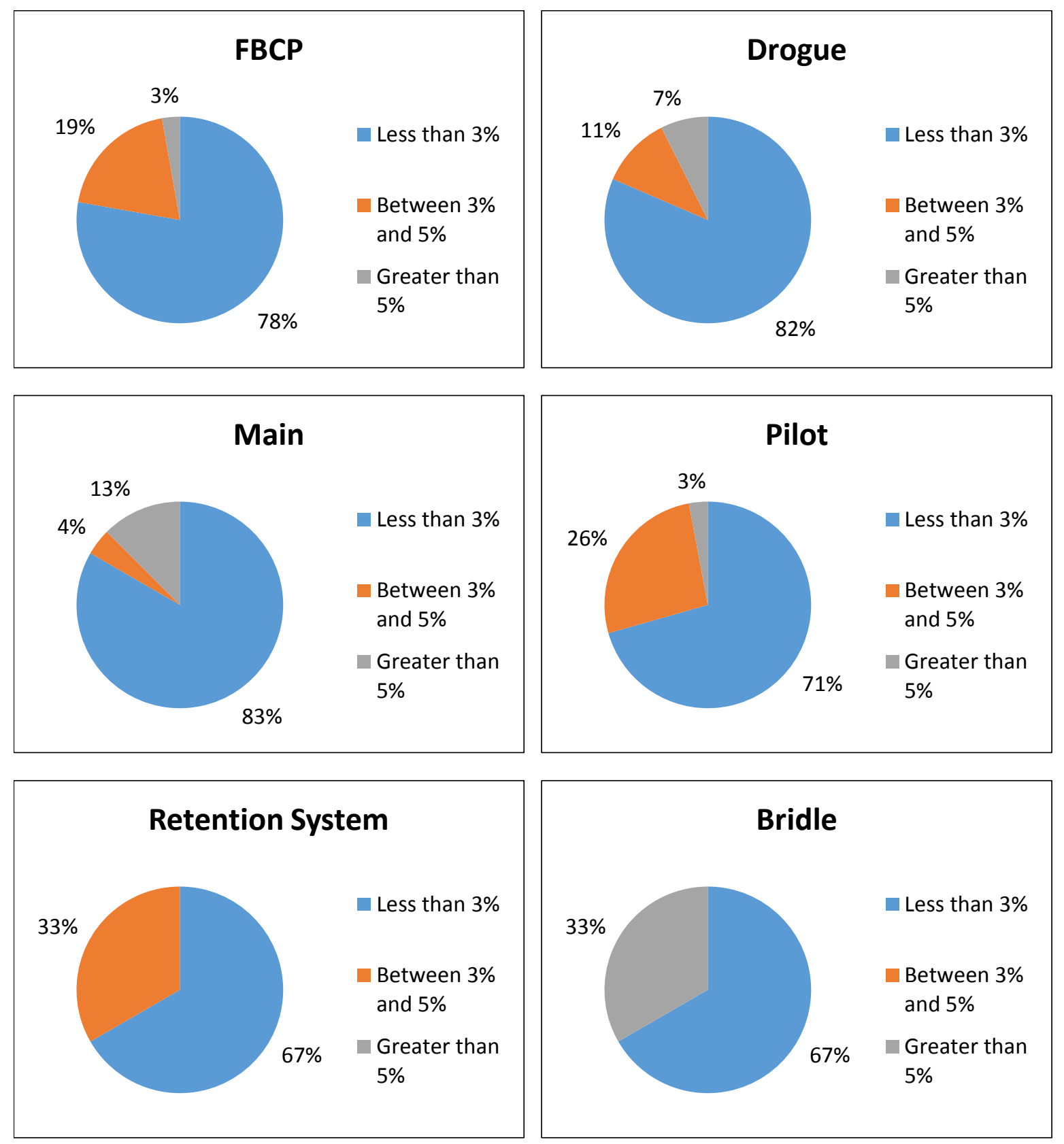

Figure 3. Percent Difference, Broken into Different Subsystems

Both the retention system and the retention release bridle have higher than average percent differences. This is because there were only three joint tests each for the retention system and the bridle, while each of the four parachutes had 15-30 joint tests; this results in a single joint test having a significant contribution to the overall results.

When comparing the results of the four parachutes to each other, both the pilot and FBCP had more test sets with large percent differences than the main and drogue. This discrepancy is partly because the pilot and the FBCP had a higher proportion of tests of the horizontal ribbons to other tests; as later explained in Section IV.B., these tests tend to have high percent differences. The drogue and main parachutes had additional tests compared to the pilot and FBCP, because of the existence of structural elements such as riser extensions, reefing lines, and reefing loops. Table 3 below shows the actual number of tests in each category: 
Table 3. Percent Difference, Broken into Different Subsystems, Tabulated Values

\begin{tabular}{|c|c|c|c|c|c|c|c|c|}
\hline Percent Difference & FBCP & Drogue & Pilot & Main & $\begin{array}{c}\text { Retention } \\
\text { System }\end{array}$ & Bridle & Total & $\begin{array}{c}\text { Percent } \\
\text { of Total }\end{array}$ \\
\hline Less than 3\% & 28 & 44 & 24 & 40 & 4 & 4 & 144 & $78 \%$ \\
\hline Between 3\% and 5\% & 7 & 6 & 9 & 2 & 2 & 0 & 26 & $14 \%$ \\
\hline Greater than 5\% & 1 & 4 & 1 & 6 & 0 & 2 & 14 & $8 \%$ \\
\hline Total & 36 & 54 & 34 & 48 & 6 & 6 & 184 & $100 \%$ \\
\hline
\end{tabular}




\section{Split by Base Component}

Figure 4 below gives the percent difference broken into different base components. All suspension line tests had Kevlar $^{\circledR}$ cord as the base material, and all radial and skirt band tests had Kevlar ${ }^{\circledR}$ webbing as the base material. The ribbon and broadcloth tests were either Nylon broadcloth (main), Nylon ribbon (drogue and pilot), or Kevlar ${ }^{\circledR}$ ribbon (FBCP). "Other" joint tests had base components such as the reefing line, vent hoop, reefing loops, and those from the retention release bridle and retention panels.
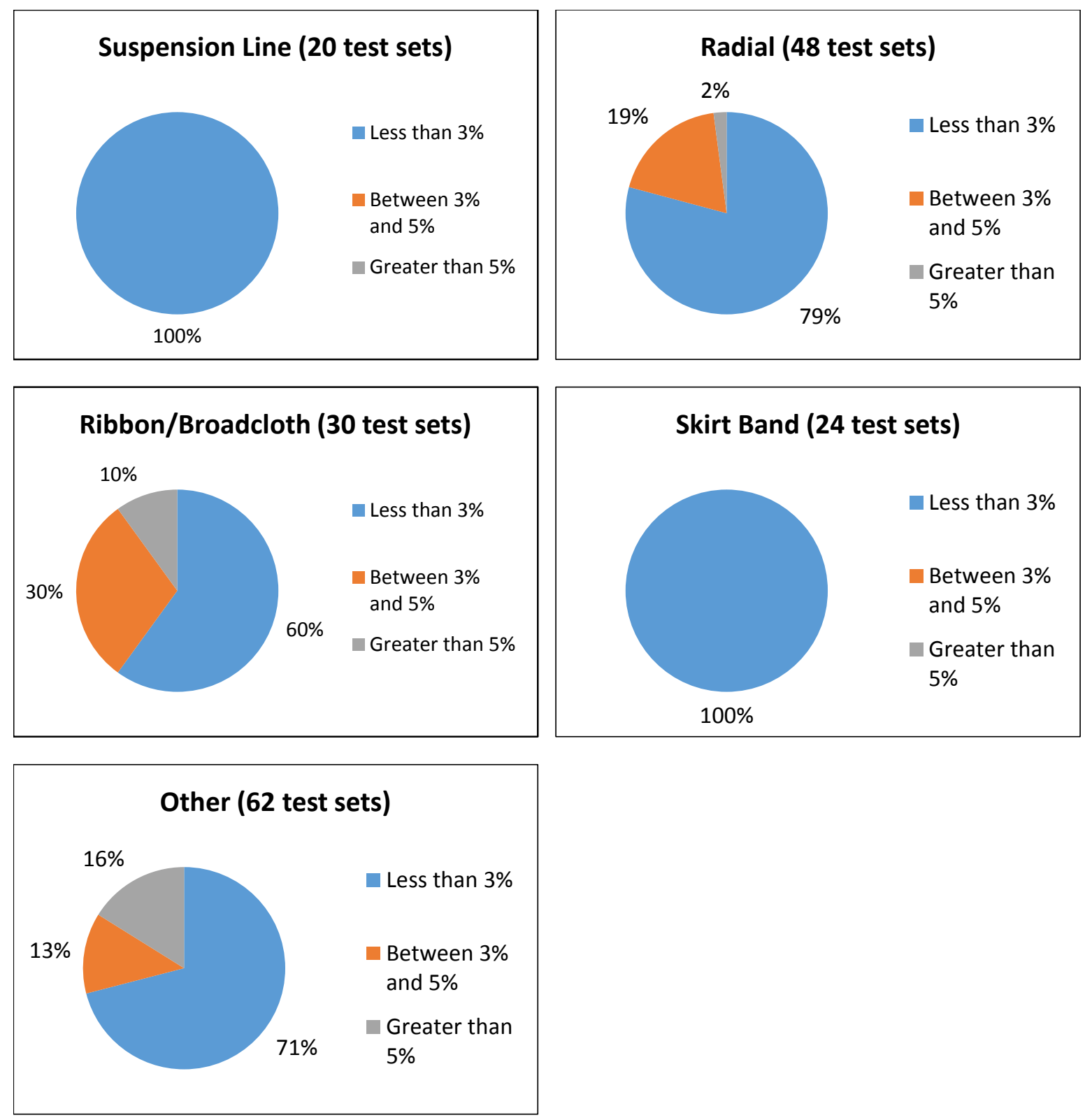

\section{Figure 4. Percent Difference, Broken into Different Base Components}

Figure 4 depicts clear differences between different structural components. Every joint test of a suspension line and a skirt band across all four parachutes had little variation between test sets. Radial joint tests had variation similar to the overall average (from Figure 2). Joint tests of ribbons or broadcloth saw more variation than average and other components. The differences seen in Figure 4 will be partially explained by Section IV, which identifies other variables which contribute to joint strength variation. 


\section{B. Confidence Level and Critical Z-Score}

As stated in Section II.C, the z-score is a measure of how many standard deviations a joint sample value is away from the average. The confidence level is how likely each joint sample value is to be below the critical z-score. See Table 4 for the applicability of the z-score to the CPAS test data:

Table 4. Confidence Level and Critical Z-Score for All Samples

\begin{tabular}{|c|c|c|c|c|}
\hline $\begin{array}{c}\text { Confidence } \\
\text { Level }\end{array}$ & $\begin{array}{c}\text { Critical Z- } \\
\text { Score }\end{array}$ & $\begin{array}{c}\text { Total Number of } \\
\text { Samples Greater than } \\
\text { Critical Z-Score }\end{array}$ & $\begin{array}{c}\text { Total Number of } \\
\text { Samples }\end{array}$ & $\begin{array}{c}\text { Percent of Samples } \\
\text { Greater than Critical Z- } \\
\text { Score }\end{array}$ \\
\hline $68.5 \%$ & 1.00 & 302 & 915 & $33.0 \%$ \\
\hline $86.8 \%$ & 1.50 & 100 & 915 & $10.9 \%$ \\
\hline $95.5 \%$ & 2.00 & 18 & 915 & $2.0 \%$ \\
\hline $98.8 \%$ & 2.50 & 0 & 915 & $0.0 \%$ \\
\hline
\end{tabular}

Table 4 shows that a confidence level of $98.8 \%$ (a critical z-score of 2.50) is required for all joint samples to be within the chosen range; with a confidence level of $86.8 \%$ (a critical z-score of 1.50 ), only $10.9 \%$ of joint samples are outside of that range.

\section{Overview of Results}

Each CPAS joint test consists of two test sets, where each test set was constructed by a separate set of operators on a separate set of sewing machines. The analysis completed on the CPAS seam and joint data shows that for the majority of joint tests, there is little difference between the two test sets- $78 \%$ of the test sets had a percent difference less than 3\% from the overall joint test average. After controlling for distribution of joint tests, it was shown that the distribution of percent differences has little variation over the six CPAS subsystems. The variation between test sets among different base components can be explained by other variables (explained more in Section IV).

Part of the reason that there are only minor variations between sets of operators and sewing machines is because of Airborne Systems's emphasis on quality manufacturing and inspection. Although there were 28 total operators who contributed to the manufacturing of the CPAS joint test, all of the joint samples and control samples are made by a detailed set of instructions. Critical steps and dimensions are inspected and verified. Additionally, individual operators are trained by qualified supervisors in order to ensure competence and understanding. With this emphasis on quality and consistency, all operators are essentially interchangeable for most tasks and uniformity is assured.

The next section will further examine and categorize those joint tests which saw large percent differences between the test sets. 


\section{Further Categorization of Joint Tests}

A. Overview of Categorization

The majority of the joint tests with high percent differences and high z-scores have one of the following attributes:

1. The breaking strength values of both the joint samples and the control samples were under $500 \mathrm{lbf}$

2. Joint samples were of a "complex" design, where "complex" is defined as having different components on either end of the joint

3. During the test, the stitches holding the base component(s) together broke, not the base component itself Figure 5 displays the breakdown of all of the joint tests into these three categories, plus the remaining joint tests:

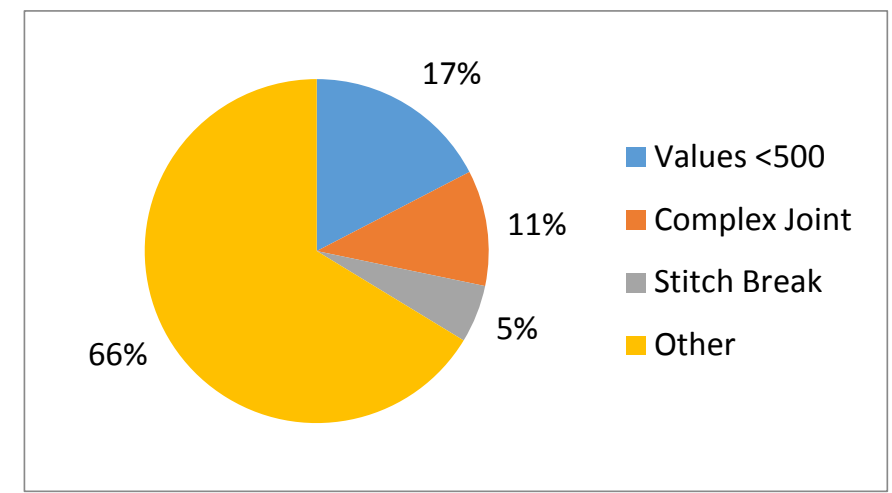

Figure 5. Data Categorization of All Joint Tests

The percent difference between the average of each test set and the average of the overall joint test was calculated for each of these joint tests in the three categories (as well as for the other remaining tests). See Figure 6:
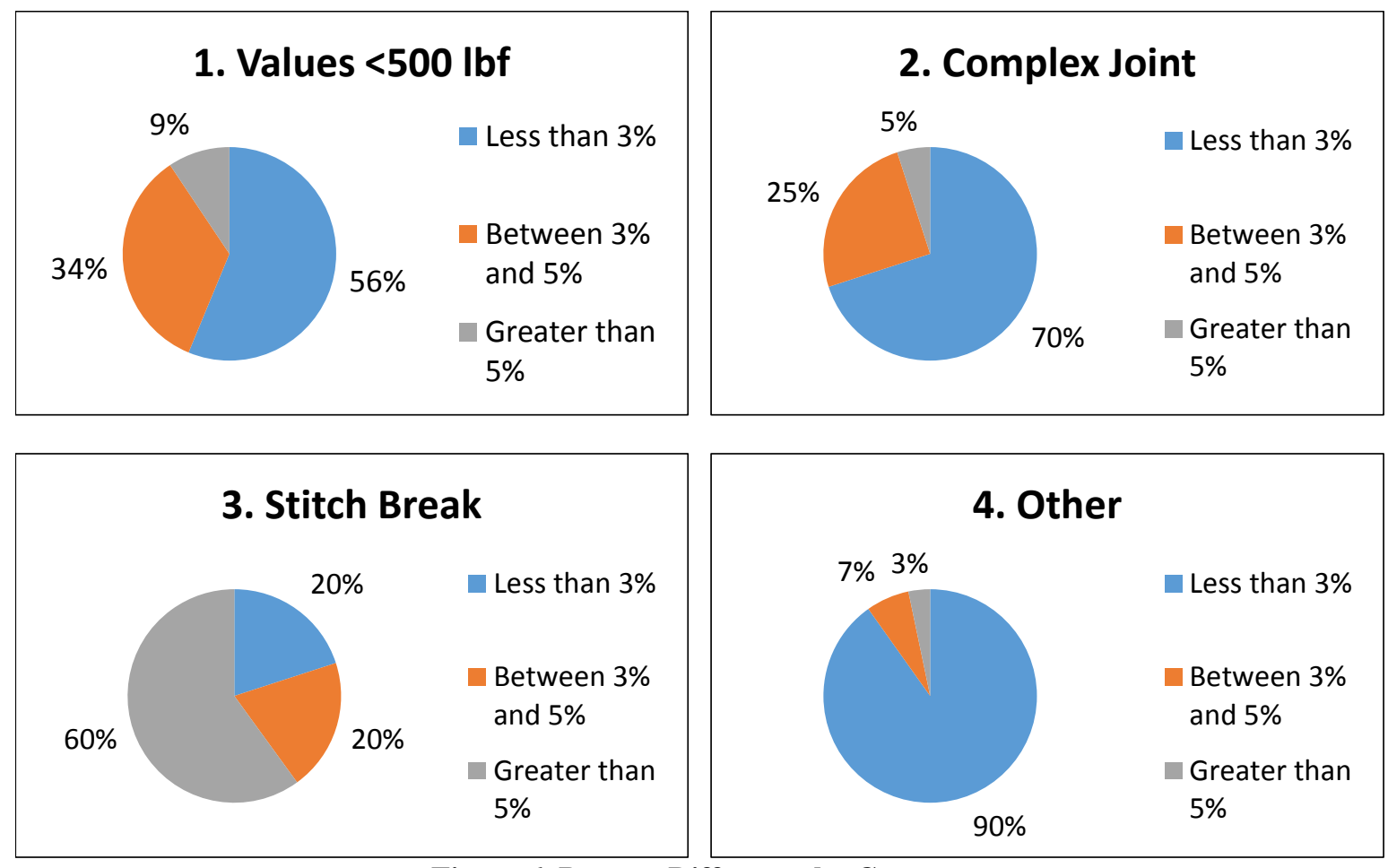

Figure 6. Percent Difference by Category 


\section{B. Joint Strength Less than $500 \mathrm{lbf}$}

Joints with tensile strengths less than $500 \mathrm{lbf}$ include all tests with horizontal ribbons (FBCP, drogue, and pilot parachutes) or broadcloth (main parachute) as the base component, as well as a few others. Using percent difference to determine the difference between the two test sets is problematic for these low strength joints. For example, the joint test for the FBCP horizontal overlap resulted in average breaking strengths that were similar to each other for the two test sets, but that still resulted in high percent differences from the average. See Table 5. While these percent differences are high compared to other joints on the same parachute, simple comparison shows that the breaking values are not that different from each other. Furthermore, this variation in strength is not enough to affect the margin of safety.

Table 5. Joint Test Results for FBCP Horizontal Overlap

\begin{tabular}{|c|c|}
\hline \multicolumn{2}{|c|}{ Breaking Strength Comparison } \\
\hline Average: Test Set 1 & $169 \mathrm{lbf}$ \\
\hline Average: Test Set 2 & $153 \mathrm{lbf}$ \\
\hline Average: Overall Joint Test & $161 \mathrm{lbf}$ \\
\hline Percent Difference: Test Set 1 to the Average & $4.9 \%$ \\
\hline Percent Difference: Test Set 2 to the Average & $5.1 \%$ \\
\hline
\end{tabular}

Omitting joints tests with breaking strength values less than $500 \mathrm{lbf}$ improves the overall distribution of percent differences, as shown in Figure 7:

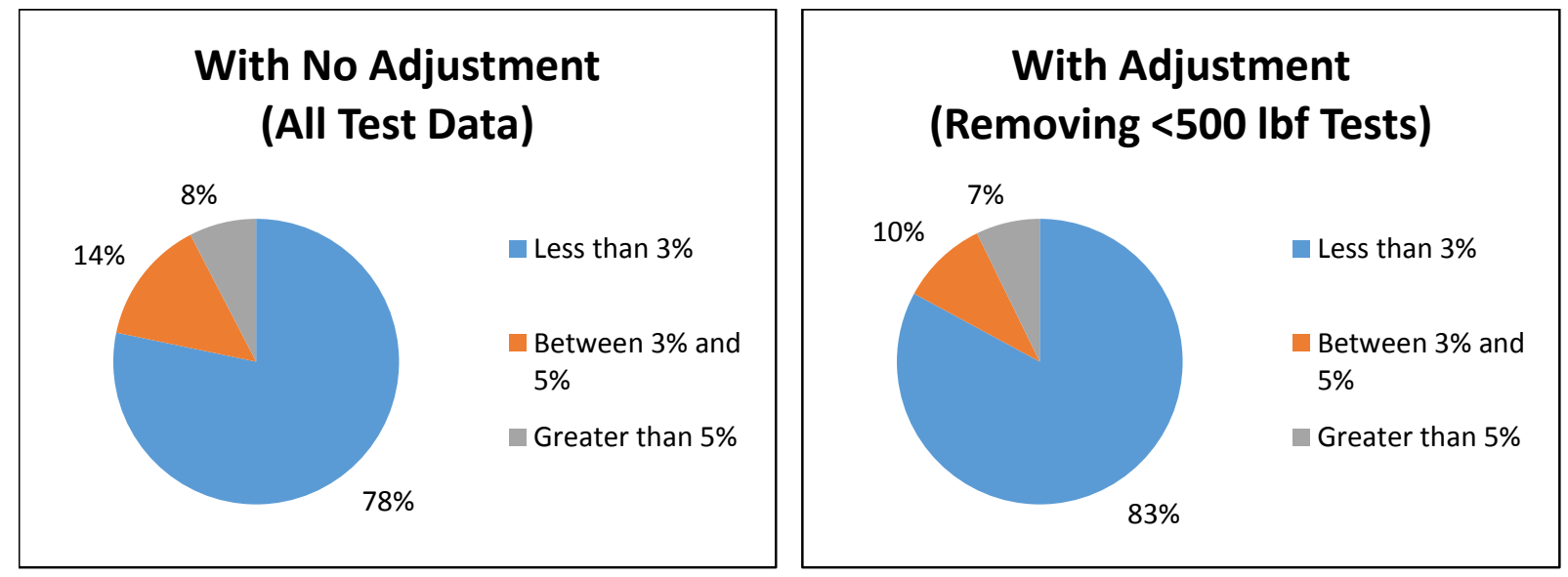

Figure 7. Percent Difference, Adjusting for Tests having Breaking Strengths < $500 \mathrm{lbf}$

\section{Complex Joints}

A complex joint design is defined as one in which there is a different component on either side of the joint, i.e. there is a different material in either jaw of the tensile testing machine. In addition to having two different base materials, these joints often also interact with multiple other components. An example is "suspension line at radial"; in addition to the connection of the suspension line with the radial, there are also interactions with the skirt band, reefing loops or rings, cutter pocket, broadcloth, and/or ribbons. These types of joints are standard for most parachutes, as load paths in a parachute often traverse multiple structural parachute elements. In order to mitigate potentially high standard deviations during a seam and joint testing program, it is recommended to either:

1. Split these joints into multiple joint tests, or

2. Increase the number of joint samples tested for these joints

The second recommendation would result in data that is more likely to group around the average, thus reducing the standard deviation. For an example of the first recommendation, the interface between the suspension line and the radial can be split into two joint tests: "radial at suspension line" and "radial at reefing loops/skirt band", as shown in Figure 8. Although this separates components into separate tests, this does not eliminate the validity of the results. 

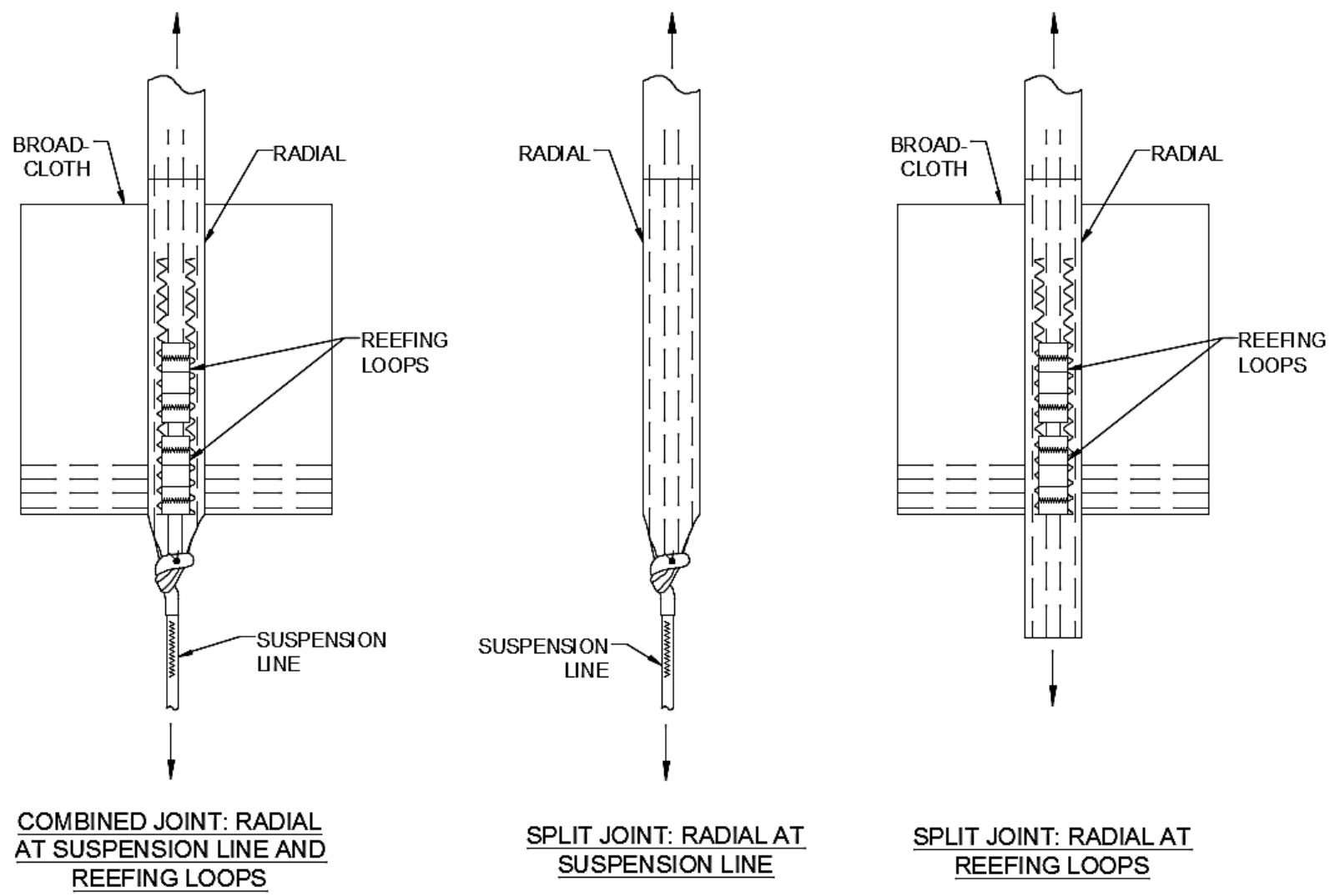

Figure 8. Splitting a Complex Joint into Two Separate Joints. The joint shown in the first image, a typical one, can be split into two separate joints (latter two images). This potentially reduces the standard deviation for each of the joint tests, which in turn increases the joint efficiency.

Since there are typically multiple joint tests for a single structural component, this method results in one more joint efficiency; the minimum joint efficiency from all of the tests is still used in the design factor calculation.

While either recommendation might result in increased cost and schedule (more joint samples to manufacture and more time required to test), there is the possibility that either would result in less variation among the joint samples; this could potentially decrease the standard deviation, leading to a higher joint efficiency. Since a higher joint efficiency leads to a higher safety margin, the official strength of that parachute element is increased.

\section{Stitch Breaks}

The majority (80\%) of the stitch-break joints on CPAS were on Kevlar ${ }^{\circledR}$ structural components. This is because $\operatorname{Kevlar}^{\circledR}$ makes up the great majority of the structural components on CPAS, as it has a better strength-to-weight ratio than Nylon (volume and weight are very important for space applications). When the stitches break instead of the base component, the stitches are the limiting factor, so the strength of the base component cannot be used in comparison to the joint sample strengths. A modified method of joint efficiency and margin of safety calculation is then implemented ${ }^{2}$. Part of the reason that joints tests with stitch breaks result in high variation is that the stitching itself is more operator- and sewing machine-dependent. The stitches per inch (SPI), exact stitch pattern length, and tension in the bobbin and needle threads are ultimately all operator-dependent, though the ranges are controlled by specification and experience. As previously stated, the design factor and margin of safety can still be calculated for these joints, though it must be through an alternate method. If there is not sufficient margin or if a stronger joint is desired, these joints can be redesigned in order to take full advantage of the strength of the base component. 


\section{E. Overview of Data when Omitting Variation-Prone Joints}

If joint tests with the three classifications from this section were omitted (breaking values less than $500 \mathrm{lbf}$, complex joints, and stitch breaks), the overall analysis of percent differences between test sets and the average vastly improves. See Figure 9.

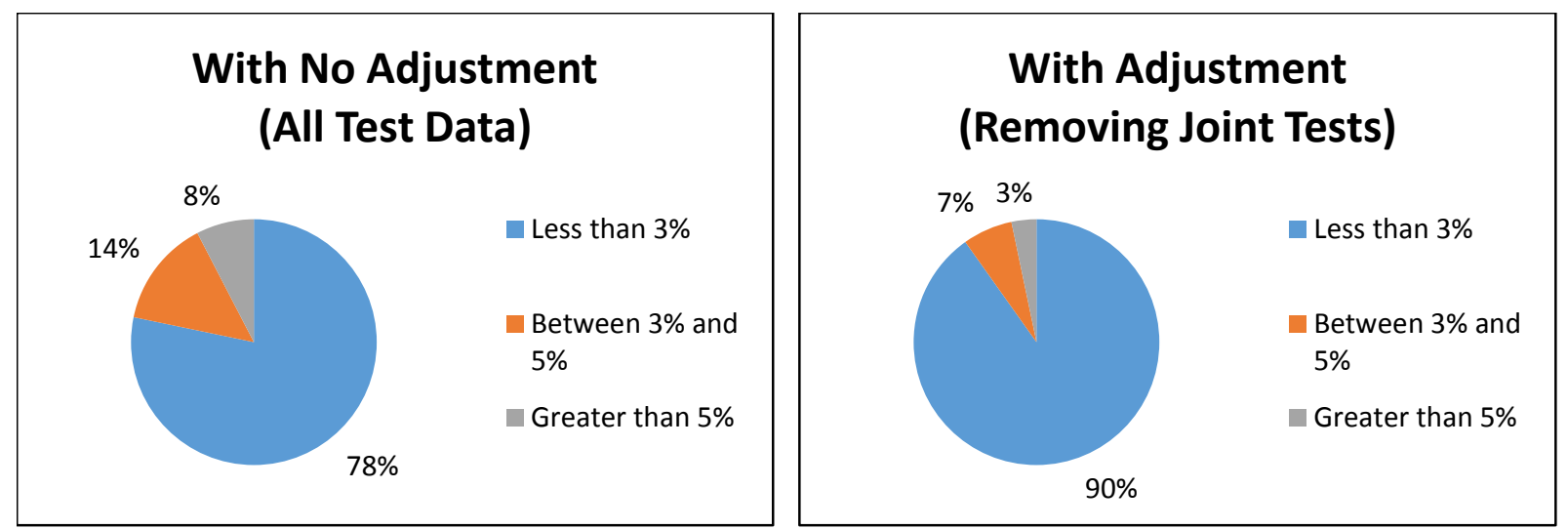

Figure 9. Percent Difference, Omitting Joint Tests. The graph on the left shows all the test data. The graph on the right omits tests that:

- Have breaking strengths less than $500 \mathrm{lbf}$

- Are classified as "complex"

- Resulted in stitch-breaks

These joint tests cannot actually be excluded from the analysis (unless the joints are redesigned), but Figure 9 illustrates the effects that these types of joints have on strength variation. While joint tests with breaking strengths less than $500 \mathrm{lbf}$ are unable to be avoided, there are methods to avoid an excess of stitch break and complex joints.

\section{F. Applicability to Different Structural Components}

The graphs in Figure 4 split up the percent difference results based on type of base component: suspension line, radial, ribbon/broadcloth, skirt band, and other. The differences in these five graphs can partially be explained using the deductions from this section. $40 \%$ of joint tests that had ribbon or broadcloth as the base component had percent differences above $3 \%$, compared to just $29 \%$ of the remaining joint tests; this is because the majority of these joints had joint sample and/or control sample breaking values $<500 \mathrm{lbf}$. Additionally, several of the radial tests were "complex" joints or consisted of numerous components; this led to slightly more occurrences of high percent differences for these joints. Suspension line and skirt band joint tests tended to be simpler and made up of fewer components, which leads to lower variations in strength.

\section{Base Material Strength Variability}

One variable not accounted for in previous sections is the strength variation inherent in the base material itself. While some variation in breaking values within a test set is based on joint design and manufacturing construction differences, a portion of it is a result of the natural strength variability in the base material. This variability is dependent on material type (Nylon, Kevlar ${ }^{\circledR}$, etc.) and construction type (weave, braid, etc.), among other things.

Each joint test on CPAS utilized 5 control samples, which are lengths of the base material with no sewing or junctions with other materials. For each set of control samples, the variation in the breaking strengths was determined by calculating the standard deviation divided by the average; dividing by the average allows for a direct comparison between materials of different strengths. Table 6 tabulates the variations from all of the sets of control samples broken during the CPAS seam and joint testing campaign: 
Table 6. Inherent Variability in Material. Except for "number of tests," each value is calculated by dividing the standard deviation by the average of the control breaking strength within that set of controls.

\begin{tabular}{|c|c|c|c|c|c|c|c|c|c|c|}
\hline Material & \multicolumn{4}{|c|}{ Nylon } & \multicolumn{5}{|c|}{ Kevlar $^{\circledR}$} & Vectran $^{\circledR}$ \\
\hline $\begin{array}{c}\text { Component } \\
\text { Type }\end{array}$ & $\begin{array}{c}\text { Tape/ } \\
\text { Webbing }\end{array}$ & Cloth & Thread & All & $\begin{array}{c}\text { Tape/ } \\
\text { Webbing }\end{array}$ & Cloth & Cord & Thread & All & Cord \\
\hline $\begin{array}{c}\text { Number of } \\
\text { Tests }\end{array}$ & 11 & 3 & 3 & 17 & 46 & 1 & 19 & 2 & 68 & 6 \\
\hline Minimum & $0.2 \%$ & $1.2 \%$ & $1.0 \%$ & $0.2 \%$ & $0.5 \%$ & $5.7 \%$ & $1.5 \%$ & $4.0 \%$ & $0.5 \%$ & $2.4 \%$ \\
\hline Maximum & $7.6 \%$ & $4.7 \%$ & $3.2 \%$ & $7.6 \%$ & $11.1 \%$ & $5.7 \%$ & $4.9 \%$ & $11.2 \%$ & $11.2 \%$ & $9.4 \%$ \\
\hline Average & $2.0 \%$ & $2.6 \%$ & $2.4 \%$ & $2.2 \%$ & $2.8 \%$ & $5.7 \%$ & $3.4 \%$ & $7.6 \%$ & $3.2 \%$ & $5.2 \%$ \\
\hline $\begin{array}{c}\text { Standard } \\
\text { Deviation }\end{array}$ & $2.0 \%$ & $1.8 \%$ & $1.3 \%$ & $1.8 \%$ & $1.7 \%$ & -- & $1.1 \%$ & $5.1 \%$ & $1.8 \%$ & $2.3 \%$ \\
\hline
\end{tabular}

Although fewer Vectran ${ }^{\circledR}$ control samples were available for comparison purposes, Vectran ${ }^{\circledR}$ had higher variations within sets of control samples than both Nylon and Kevlar ${ }^{\circledR}$. This is partly a consequence of material properties; Nylon tends to elongate $\sim 30 \%$ at failure, while $\operatorname{Kevlar}^{\circledR}$ and Vectran ${ }^{\circledR}$ elongate closer to $\sim 3 \%$. The ability to elongate allows for better load sharing within the material, leading to more consistent breaking strengths. Across the three material construction sub-categories (tape/webbing, cloth, and thread) that Nylon and Kevlar ${ }^{\circledR}$ share, Nylon showed less variation within control sample sets than Kevlar ${ }^{\circledR}$. In both Nylon and Kevlar ${ }^{\circledR}$ sample sets, tape and webbing had the least variation in breaking strength, while thread and cloth tended to have the most variation.

\section{Effect of Number of Joint Samples}

The number of joint samples within each joint test is typically a requirement levied by the customer and/or is driven by standard practices and schedule/cost. In order to assess the effects of the number of joint samples, the seam and joint test results from three recent and similar programs were compared to each other. Within each program, the difference between the standard and mean efficiency was calculated for each joint test. This calculation is a measure of the variation within a joint test (see Section II.B for more detail). The results are tabulated below in Table 7:

Table 7. Effects of Number of Joint Samples, as Seen by Multiple Programs.

\begin{tabular}{|c|c|c|c|c|}
\hline \multicolumn{2}{|c|}{ Program } & CPAS & Program B & Program C \\
\hline Number of Joint Samples in a Joint Test & $10(5+5)$ & 10 & 5 \\
\hline \multicolumn{2}{|c|}{ Number of Joint Tests Analyzed } & 89 & 42 & 51 \\
\hline \multirow{3}{*}{$\begin{array}{c}\text { Standard } \\
\text { Mean } \\
\text { Efficiency }\end{array}$} & Average & $4.0 \%$ & $3.5 \%$ & $4.1 \%$ \\
\cline { 2 - 5 } & Standard Deviation & $1.7 \%$ & $1.5 \%$ & $1.8 \%$ \\
\cline { 2 - 5 } & Avg + StDev & $5.7 \%$ & $5.0 \%$ & $5.8 \%$ \\
\cline { 2 - 5 } & Avg - StDev & $2.3 \%$ & $2.0 \%$ & $2.3 \%$ \\
\hline
\end{tabular}

CPAS, as previously stated, required ten samples split into two different test sets. Program B used ten samples per joint test, all from the same operator and sewing machine. Program $C$ used only 5 samples per joint test, all from the same operator and sewing machine.

The differences between the three programs, though slight, indicate that the number and configuration of joint samples may have an effect on joint test variation. Program B, which had the most number of samples built by the same sets of operators and sewing machines, saw a difference of 2-5\% between standard and mean efficiency. CPAS, which split the ten samples into two sets, and Program C, which only had 5 samples, saw a difference of 2.3-5.8\%. While this is not conclusive, it does present an argument for constructing a seam and joint test program to have more samples from a single set of operators and sewing machines. When comparing Program B to Program C, it can be seen that having a single operator manufacture more samples (10 instead of 5) tends to generate a central tendency in the variation of results, as the standard deviation decreased from $1.8 \%$ to $1.5 \%$. 


\section{Summary and Recommendations}

There are numerous areas that affect material and joint strength. Some of these have a minor effect on joint strength variability and some have a major effect. The following offers guidelines and recommendations for future joint design and seam and joint testing campaigns:

1. Multiple operators and sewing machines: the requirement for implementing two or more sets of operators and sewing machines is not necessary if the overall standards for quality of training, manufacturing, and inspection are high. If all joint samples and control samples are manufactured using the same lots of materials and the same steps, there should be little variation between operators/sewing machines. This requirement requires more time and cost, with little added benefit or understanding.

2. Complex joints: While complex joints exist on every parachute, strategies can be implemented during seam and joint testing in order to reduce strength variability among the joint samples. A recommended alternative to a single joint test is to split up these complex joints into multiple simple tests that have fewer components. Additionally, more samples can be ordered for these joints in order to potentially reduce the standard deviation.

3. Stitch break joints: the most operator- and machine-dependent component is the stitching. When joint samples break the stitching instead of the base component, the variation in breaking strength becomes operator- and machine-dependent. Additionally, stitch breaks indicate that the full strength of the base material is not being utilized, although positive margin may still exist. If this type of break occurs during a joint test, it is recommended to redesign the stitch pattern to be stronger.

4. Number of joint samples: a larger number of control samples and/or joint samples could potentially reduce the standard deviation within a joint test. While this has to be balanced by cost and schedule, a larger number of joint samples (10-20) is particularly recommended in situations where larger standard deviations result in low standard efficiency values.

\section{Conclusion}

The objective of this paper was to use the data and results from the CPAS seam and joint testing program to offer comments and make recommendations for future programs. The previous section offers advice for future programs, with recommendations such as:

- Avoid requiring multiple sets of operators and sewing machines for a single joint test

- Split complex joints into more than one joint test

- Perform sufficient analysis on each joint to reduce the likelihood of the stitches breaking. If the stitches do break and the resulting safety margin is low, it is recommended to redesign the joints

- Increase number of joint samples to the extent possible (10-20 recommended), in particular for complex joints or those with high standard deviations

Variation in joint strength can be caused by many things, some of which are listed above. If these scenarios are avoided during a seam and joint testing program, not only can cost and time often be saved, but higher joint efficiencies can be achieved. Higher joint efficiencies lead to higher margins of safety, which might allow for weaker and lighter structural components, saving parachute and system weight and volume. Some parachute structural elements and joints will naturally have large variations in strength, whether it is due to material choice, complexity of design, strength of materials, etc. But with the analysis presented and the recommendations made in this paper, seam and joint testing campaigns can become more effective and representative.

\section{Acknowledgments}

Thanks to CPAS for a plethora of seam and joint data. And very special thanks to all of the engineers who spent countless hours making drawings, breaking samples, and entering data!

\section{References}

${ }^{1}$ Knacke, T. W., Parachute Recovery Systems Design Manual, $1^{\text {st }}$ ed., Para Publishing, Santa Barbara, California, 1992.

${ }^{2}$ Mollmann, Catherine, "Determination of Parachute Joint Factors using Seam and Joint Testing," AIAA 2015$2125,2015$.

385-1898B, "CPAS Seam and Joint Test Report", Airborne Systems North America, February 2016. 